\title{
Lamb wave transducers made of piezoelectric macro-fiber composite
}

\author{
Michał Mańka ${ }^{1, * \dagger}{ }^{\dagger}$, Mateusz Rosiek ${ }^{1}$, Adam Martowicz ${ }^{1}$, Tadeusz Stepinski ${ }^{1,2}$ and \\ Tadeusz Uhl ${ }^{1}$ \\ ${ }^{1}$ Faculty of Mechanical Engineering and Robotics, Department of Robotics and Mechatronics, AGH University of Science and \\ Technology, al. A. Mickiewicza 30, 30-059 Krakow, Poland \\ ${ }^{2}$ Signals and Systems, Uppsala Universitet, P.O. Box 534, SE-751 21 Uppsala, Sweden
}

\begin{abstract}
During recent years, an intensive research activity concerning the application of Lamb waves (LWs) for SHM has been observed. LWs may be generated and sensed using different types of transducers, and their selection is essential for the SHM system's performance. Results of the investigation of three types of transducers based on macro-fiber composite (MFC) are presented in this paper; two types of commercially available MFC actuators are compared with a novel type of custom-designed interdigital transducer also based on the MFC substrate. After a short presentation of the piezoelectric transducer designed for SHM applications, details concerning the proposed interdigital transducer design are provided. Beampatterns of the investigated transducers are first compared using numerical FEM simulations, and next, the numerically obtained beampatterns are verified experimentally using laser vibrometry. In the final part of this paper, advantages and disadvantages of the investigated transducers are discussed. Copyright (C) 2012 John Wiley \& Sons, Ltd.
\end{abstract}

Received 22 February 2012; Revised 19 August 2012; Accepted 7 September 2012

KEY WORDS: structural health monitoring, Lamb waves, piezoelectric transducers, interdigital transducers, macro-fiber composites

\section{INTRODUCTION}

Ultrasonic guided waves (GWs) are structure-borne elastic waves that propagate along the structure confined and guided by its geometric boundaries. During their propagation, GWs interact with geometric discontinuities and produce reflected waves, which can be detected by appropriate transducers that are also often used for generating the incident GWs. Despite their complicated nature, GWs have gained importance in SHM applications, primarily because of their ability to propagate over long distances with little attenuation. Their additional advantage is selective sensitivity of different GW modes to a variety of structural defects. For instance, the fundamental symmetric (S0) Lamb mode, which has dominant in-plane components, is sensitive to the through-the-thickness damage, whereas the A0 mode is better suited for detecting surface damage because of its dominant out-of-plane component. A review and state of the art of the SHM systems was presented by Raghavan and Cesnik [1].

Lamb wave (LW) transducers used in SHM should be small and light to be integrated into the monitored structure without influence on its behavior. These requirements are especially important for applications where aerodynamic or hydrodynamic properties of the structure should remain unchanged [2]. Additionally, to reduce the system cost, each LW transducer should cover a large area of the monitored structure, and its cost should be low to make the system economically justified.

*Correspondence to: Michał Mańka, Faculty of Mechanical Engineering and Robotics, Department of Robotics and Mechatronics, AGH University of Science and Technology, al. A. Mickiewicza 30, 30-059 Krakow, Poland.

†E-mail: mmanka@agh.edu.pl 
This paper is concerned with piezoelectric transducers capable of generating and sensing LWs in SHM systems with the aim of efficient damage interrogation. LWs can be excited in planar structures by using different devices, for example, ultrasonic angle beam transducers [4], electromagnetic acoustic transducers [3-5], and laser ultrasound systems [6-9]. Many of those transducers do not comply with the demands of SHM systems because they are bulky (e.g., angle beam transducers), they are brittle (piezo-wafers), or they generate waves with low energy (e.g., Capacitive micromachined ultrasonic transducers and electromagnetic acoustic transducers).

Piezoelectric transducers made of lead zirconate titanate (PZT) ceramics are affordable, and their sizes are relatively small compared with the monitored structure. Thin and preferably flexible piezoelectric patches capable of generating intensive LWs with high directionality are the most suitable transducers for SHM systems. Two types of such transducers can be distinguished from the point of flexibility: the inflexible piezoceramic patches with uniform electrodes and the flexible patches made of piezocomposites, for example active fiber composites (AFCs) [10-13] and macro-fiber composites (MFCs) [14-19]. The AFC and MFC devices are usually optimized as actuators and provided with dense comb-like electrodes. It appears, however, that sparse comb-like electrodes used in interdigital transducers (IDTs) [20,21] create very interesting properties of mode selectivity. Feasibility of the IDT for generation of LWs in application to structure monitoring was investigated by Monkhouse et al. [22,23]. They used polyvinylidene difluoride (PVDF) as piezoelectric material to manufacture and investigate IDTs with different topologies for SHM applications. PVDF is a very flexible and cheap material; however, it exhibits considerably weaker piezoelectricity than PZT ceramics. Moreover, it cannot be used at high temperatures, which may preclude its use in some structures.

This paper is focused on the transducer that may be mounted on the long curved or deformable thin-walled structures (i.e., blades of wind turbines or airplanes wings). Transducers mounted on such structure should be elastic or small enough to avoid damage from the structure deformations. Moreover, they should be able to generate directional wave to avoid reflections from the structures' boundaries.

In this paper, the authors investigate three different transducers made of MFC substrate, two commercially available actuators with dense comb electrodes, and a custom-designed IDT with double-sided sparse interdigital electrodes. Beampatterns of all the three transducers are first calculated using numerical simulation and are then compared with those measured using laser vibrometer. The transducers' beampatterns are compared in terms of the main lobe width and side lobe level. Mode selectivity is also investigated using time and frequency analysis of the generated waves. The aim of this work is to find which of the investigated transducers has the best directivity and mode selectivity.

This paper is organized as follows. Section 2 contains a short presentation of the piezoelectric transducers with focus on the SHM implementation. Design process of the IDT is shortly described in Section 3. The results of the numerical simulations are reported in Section 4, and they are compared with the experimental results in Section 5. Finally, Section 6 presents the authors' conclusions where benefits of the MFC-based IDT are discussed.

\section{PIEZOELECTRIC TRANSDUCERS FOR SHM}

\subsection{Piezoceramic patches}

Piezoelectric elements made of PZT ceramics are commonly used as transducers for LW generation in SHM applications [24-28]. Typical PZT transducers used for LW generation are built as piezoelectric patches attached to the inspected structure. Single PZT transducer generates omnidirectional wave with relatively high amplitude. Frequency, mode selectivity, and main directions of propagation of that wave are strongly related to the excitation frequency and patch shape [29]. Multiple transducers forming ultrasonic phased arrays can be used to achieve directionality of the generated waves [30,31]; however, complex electronics for signal generation and reception is required [32,33]. Usually, the size of the piezoceramic patches is below or in the range of $10 \mathrm{~mm}$, and in this case, the fragility of the PZT transducer is not the major drawback. However, if PZT layer is used for larger transducers in the range of tens of millimeters (e.g., IDT), then they can be easily damaged during integrating process or by the structure deformation in its normal operation condition. Moreover, in the case of monitoring the thin and deformable structures, stiff PZT patch can cause local increase of rigidity. 


\subsection{Transducers made of piezoelectric fiber composite}

Recently, two types of fiber piezoelectric composites suitable for patch transducers have been developed: the AFCs and the MFCs. The AFCs have been developed by Bent and Hagood from Massachusetts Institute of Technology (MIT) [10]. The structure of an AFC transducer is shown in Figure 1(a). It consists of three main layers: one layer of piezoceramic fibers embedded in an epoxy matrix and sandwiched between two sets of interdigital electrodes [11]. The piezoceramic fibers are produced through an injection molding process-PZT powder mixed with the wax binder is heated to achieve the specified viscosity and injected at high pressure into a cooled mold [12]. When dense comb-like electrodes are placed on the top and bottom of the fibers, a transducer is produced. Dense comb-like patterns allow for generating directional wave, and epoxy layers make those transducers flexible [13].

Macro-fiber composite transducers, originally invented by NASA [14], are essentially the cost-effective version of the AFC. The structure of a MFC transducer is similar to that of the AFC and also consists of three main layers as shown in Figure 1(b).

The main difference between the AFC and the MFC is the shape of the fibers; the AFC's fibers are round, whereas the MFC's are rectangular. The piezoelectric fibers, located in the central layer (active layer) of the MFC transducer, are diced from piezoceramic wafers [15]. The top and bottom layers of the transducer consist of electrode patterns. The electrode pattern may vary with the type of the piezoelectric effect used for wave generation or with the transducer's shape.

Two main types of MFC transducers are shown in Figure 2 [29]. The first type, illustrated by Figure 2(a), uses the d31 effect in the piezoelectric fibers with voltage applied through the fibers' thickness. The second type exploits the d33 effect with voltage applied along the fiber's length (Figure 2(b)). The arrows in Figure 2 show the direction of applied electric field.

Similarly to the AFC, the MFC transducers generate the directional GWs, and the use of piezocomposites makes the transducers flexible.

\subsection{Description and design principles of the interdigital transducers}

Devices made of piezoelectric fiber composite with dense interdigital electrode patterns have been used mainly as general-purpose actuators that are not optimized for any operating frequency. IDTs,
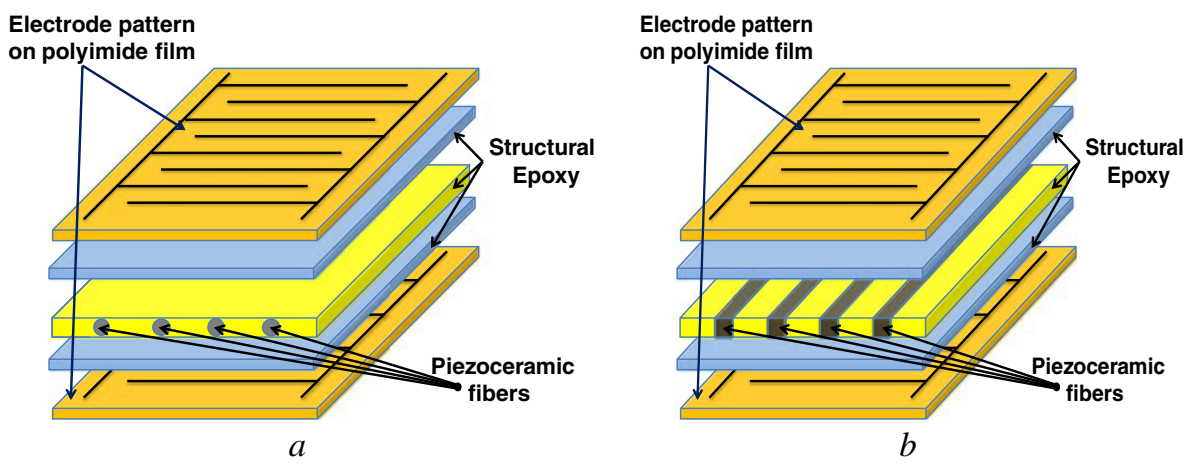

Figure 1. Structure of the (a) AFC and (b) MFC transducers.
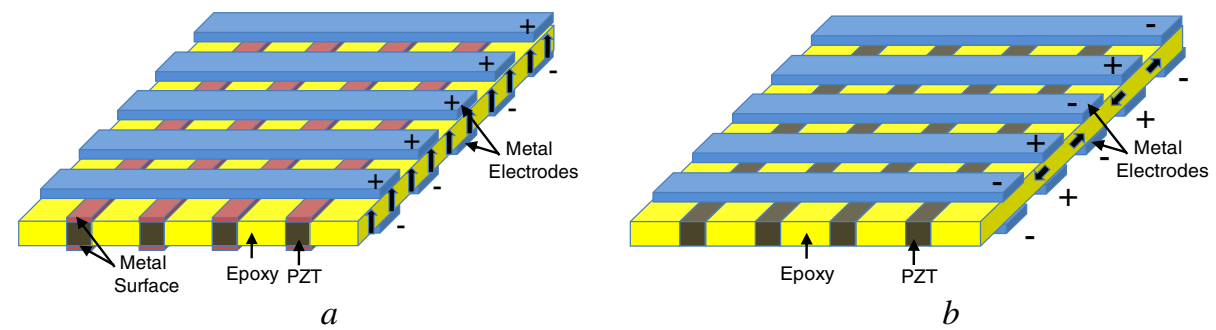

Figure 2. Examples of the MFC types: (a) d31effect and (b) d33 effect. 
however, if used for generating and sensing LWs, can be designed to excite modes with a specific wavelength. By adjusting finger spacing in the comb electrodes accordingly to the specific wavelength in the inspected plate, the transducer will become a mode-selective filter. This LW transducer type has been first created using PVDF [22,23] and, very recently, using piezoceramic composite [33,34]. The origin of IDTs can be traced back to the surface acoustic wave (SAW) devices [21]. However, the IDTs designed for LWs operate at frequencies much lower than $1 \mathrm{MHz}$, whereas the SAW devices are designed for the frequencies in the range of tens of megahertz to several gigahertz. A typical IDT, shown in Figure 3, consists of three layers: the bottom and top electrodes are separated by the piezoelectric layer.

The piezoelectric layer may be made of piezoelectric polymer (i.e., PVDF [35-37]), piezoceramic [38], or piezoceramic composite [14,39]. Properties of the piezoelectric layer determine transducer features such as elasticity, maximal energy, and frequency of the generated waves. The most significant feature of IDT transducers is the electrode pattern that has a comb/finger-like shape. Contrary to MFC actuators, IDTs are designed for the excitation of the specific, nominal wavelength that is determined by the distance between phase electrodes (finger separation) [22,23]. Similarly to AFC and MFC, IDTs generate bidirectional waves [40,41]. Signal is generated in the direction perpendicular to the finger electrodes, and the wave divergence (the main lobe width) depends on the number of fingers and their length.

Two main types of IDT transducers can be distinguished depending on the electrode pattern: the traditional single-sided IDT (IDT-SS) and the double-sided IDT (IDT-DS) [21].

Generic IDT-SS, presented in Figure 3, has comb-shaped electrodes only on one side of the piezoelectric substrate, whereas the second side is covered by the ground plate electrode. Three wires are required to connect electrical signals to the electrodes (two opposite phases and ground), which means that antisymmetric sources of the phase signals (e.g., sine bursts) have to be used to supply the interdigital electrodes.

The IDT-DS, shown in Figure 4, has both sides covered by the interdigital electrodes. To simplify the wiring, the top and bottom electrodes are connected in pairs so that the opposite phase electrodes are placed over each other. This design requires only two wires and does not need antisymmetric signal sources. Moreover, the amplitude of the GWs generated by IDT-DS can be higher than that generated by IDT-SS [21].

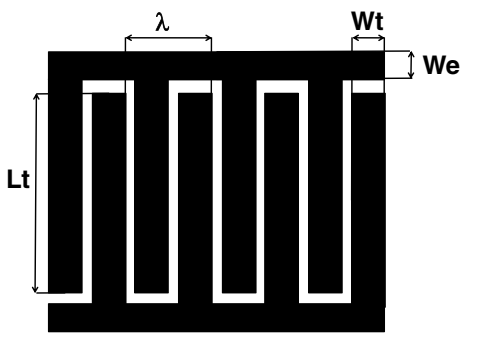

$a$

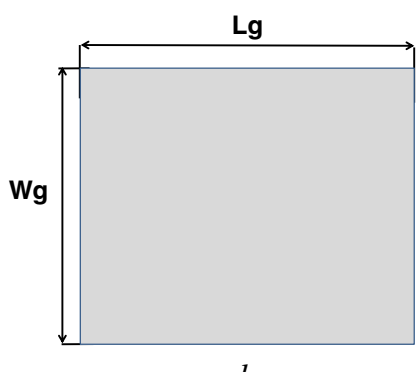

$b$

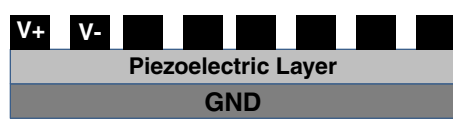
GND

$c$

Figure 3. Structure of the single- sided IDT: (a) top electrode, (b) bottom electrode, and (c) cross section.

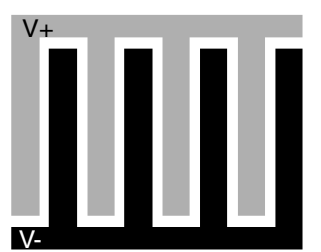

$a$

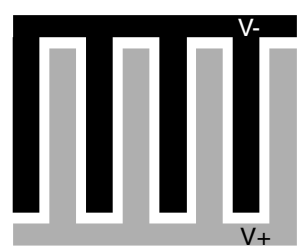

$b$

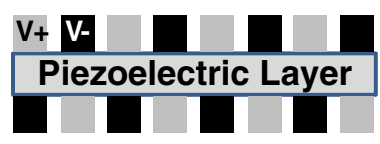

$c$

Figure 4. Electrode layout in the double-sided IDT: (a) top electrode, (b) bottom electrode, and (c) cross section. 


\section{THE MACRO-FIBER COMPOSITE INTERDIGITAL TRANSDUCER}

Motivated by the specific needs of SHM applications for long, thin, and deformable structures, this paper is focused on flat and flexible transducers that are capable of generating directional waves and can be easily integrated with monitored structures. Transducers based on AFC and MFC have similar properties and offer the desired advantages; however, because MFC is commercially available, it was chosen for investigation. Compared with monolithic PZT transducers, MFC offers increased flexibility and durability, which makes it an attractive material for a structurally integrated GW sensor. The specific aim in this paper is to compare properties of the commercially available MFC actuators with dense electrodes (Smart Material Corp., Sarasota, Fl, USA) with that of MFC-based IDT provided with comb electrodes designed for a specific wavelength.

The IDTs presented in this paper are designed to excite the A0 mode in a 4-mm-thick aluminum plate. This mode exists at a broad band of frequencies; however, the most useful for SHM applications are the frequency bands characterized by low dispersion, for example the specific frequency band for which the excited waves travel with approximately constant speed.

This situation occurs at the points where group velocity is either stationary or almost stationary with respect to frequency, which takes place at the maxima indicated by dots at the dispersion plot shown in Figure 5. In the studied case, the maximum of the group velocity of the A0 mode in a 4-mm-thick aluminum plate exists at $329 \mathrm{kHz}$, and this frequency was chosen for IDT design.

Two standard commercially available MFC transducers were chosen for the tests reported here (the P1 with d33 effect and the P2 with d31 effect), both provided by Smart Material GmbH, Dresden, Germany, who also provided a number of MFC-based IDTs with electrode combs designed by us. Dimensions of the tested transducers are presented in Table I.

The IDT investigated during performed tests has dimensions close to those of the MFC transducers. The IDT-DS was chosen for the tests because of the higher amplitude of the excited wave and simpler wiring. The transducer design process was presented in the authors' previous publications $[33,34]$. The layout and the overall dimensions of the designed transducer (further referred to as the IDT-DS4) are shown in Figure 6.

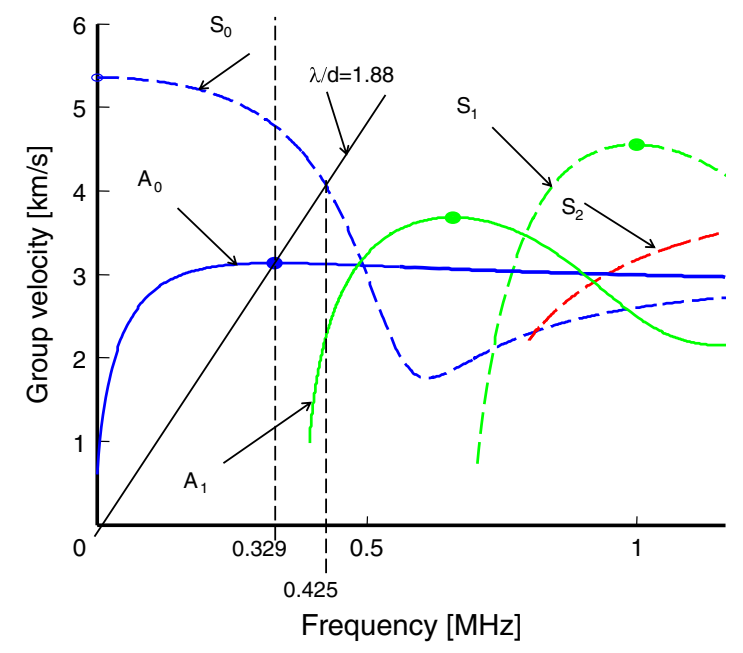

Figure 5. Group velocity of a 4-mm-thick aluminum plate.

Table I. Geometric parameters of the transducers used during experiments.

\begin{tabular}{lccc}
\hline Transducer type & MFC-M2814-P2 (d31 effect) & MFC-M2814-P1 (d33 effect) & IDT-DS4 (d31 effect) \\
\hline Active length $(\mathrm{mm})$ & 28 & 28 & 28.2 \\
Active width $(\mathrm{mm})$ & 14 & 14 & 15 \\
\hline
\end{tabular}




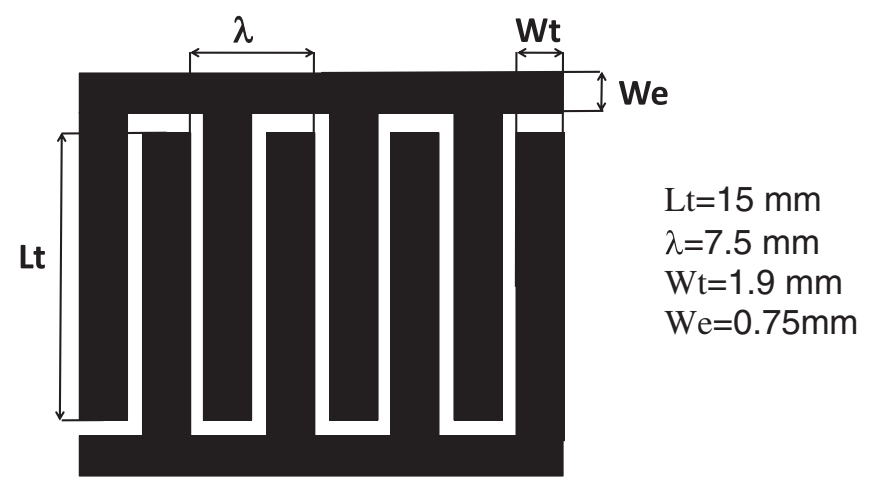

Figure 6. Top/bottom electrodes layout and dimensions.

Properties of the piezocomposite are similar in all investigated transducers (fibers $0.5 \times 0.2 \mathrm{~mm}$ ). The electrodes on both sides of the transducers are made of 0.18-mm-thick copper layer. A three-dimensional model and a photograph of the manufactured IDT are presented in Figure 7.

\section{NUMERICAL SIMULATIONS}

Numerical simulations were performed to indentify properties of the designed IDT-DS4 and the MFC transducers chosen for comparison. Numerical models of the transducers and aluminum plate were created in ANSYS Multiphysics software (ANSYS Inc., Canonsburg, PA, USA). Each of the transducers was placed centrally on a 4-mm-thick aluminum plate with dimensions $500 \times 500 \mathrm{~mm}$. A model of the structure was built using 20-node brick finite elements (FEs). Fully coupled transient analysis was performed to simulate piezoelectric effect. The transducers were excited with electrical signals (five-cycle tone burst modulated with Hanning window, amplitude $100 \mathrm{~V}_{\mathrm{p}-\mathrm{p}}$ ) as shown in Figure 8. GW propagation was simulated in the time interval $80 \mu \mathrm{s}$.

For the tests, three excitation frequencies were chosen: $330 \mathrm{kHz}$, which is the nominal frequency for the designed IDT (maximum on the dispersion plot for the A0 mode in a 4-mm-thick aluminum plate); $425 \mathrm{kHz}$, the frequency for which the S0 mode's wavelength is equal to $7.5 \mathrm{~mm}$ (finger separation); and $100 \mathrm{kHz}$, which is the low frequency excitation to verify the behavior of the tested transducers at low frequencies.

Averaged values of the MFC parameters, provided by Smart Material, were used as the properties of the piezoelectric layer (Tables A1 and A2).

\subsection{Beampattern calculation}

The response surface method (RSM) $[42,43]$ was applied to calculate beampatterns in all simulations performed to analyze the dynamic properties of the modeled piezoelectric transducers. During the FE simulations, rectangular FEs were used, and it was impossible to evaluate the amplitude directly on the radius of $150 \mathrm{~mm}$. The RSM enabled finding approximate maximal nodal velocity for all the

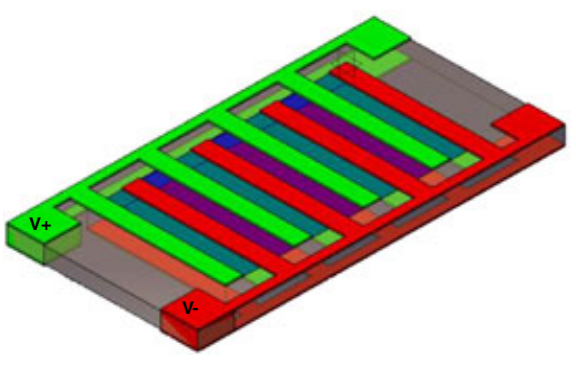

$a$

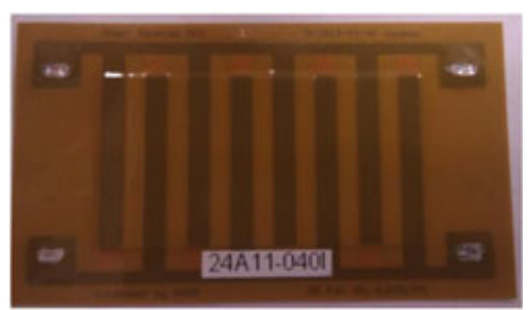

$b$

Figure 7. The IDT-DS4 used in the tests: (a) 3D schematic and (b) manufactured prototype. 


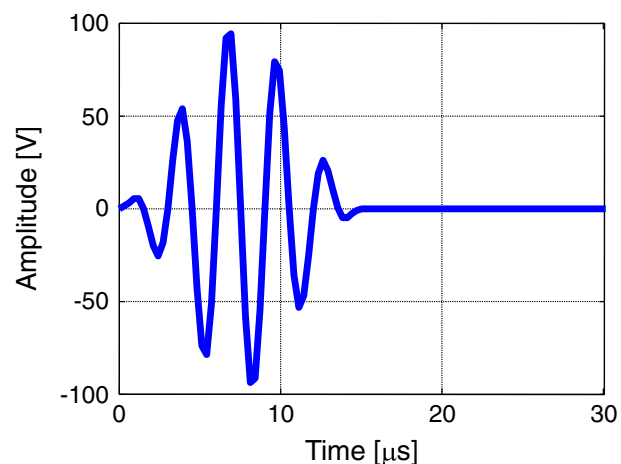

$a$

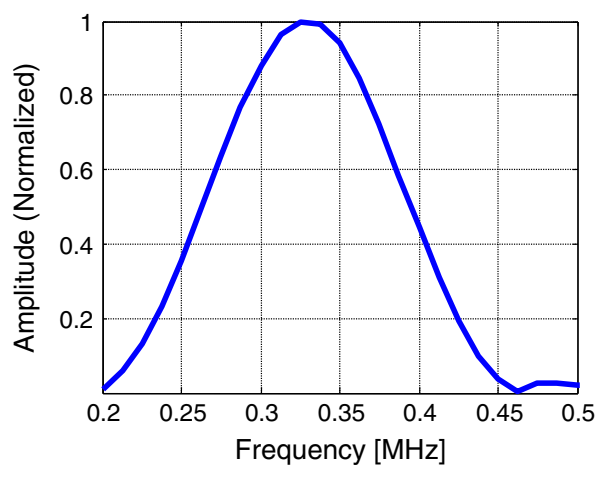

$b$

Figure 8. Excitation signal for $330 \mathrm{kHz}$ : (a) time plot and (b) frequency spectrum.

measurement points on desired radius by using a set of nodes in the FE mesh located in the close vicinity to the area with the radius of $150 \mathrm{~mm}$. Transformation of coordinates from the Euclidean to polar system was performed for the selected nodes to minimize geometric irregularities of the RSM input domain.

The Hilbert transform was used to determine envelopes for time history plots of the vertical nodal velocity for all selected nodes. Finally, the maximal value for each envelope was found and assumed to represent the nodal velocity of the generated mode (either symmetric or antisymmetric). This data was used as an input for the RSM application. An example snapshot for a node is presented in Figure 9(a) for the IDT-DS4 excited with $100 \mathrm{kHz}$.

Polynomial metamodel was applied to approximate the relationship between input parameters (normalized radius and angle) and the maximal value of the envelope. The least squares method was applied to determine the coefficients of 141 regressors by the use of 1405 known values of envelope extremes. An example of the response surface generated for the IDT-DS4 excited with $100 \mathrm{kHz}$ is presented in Figure 9(b). The normalized values of angle and radius from the interval $[-1,1]$ represent the variation $\left[-90^{\circ}, 90^{\circ}\right]$ and $[148 \mathrm{~mm}, 152 \mathrm{~mm}]$, respectively.

\subsection{Numerical results obtained for the MFC-P2 with d31 effect}

Results obtained from the numerical simulations performed for the MFC-M2814-P2 transducer presented in Figure 10 show the transducer's beampatterns for different excitation frequencies. It can be seen that the GWs generated by that transducer propagate in four perpendicular directions simultaneously for all considered frequencies. All beampatterns, however, have relatively wide lobes, which can be observed in Figures 11-13, where besides the shape of the calculated beampatterns, the calculated divergence angles are presented. Divergence angle, also known as main lobe width, is calculated as $-6 \mathrm{~dB}$ amplitude drop for each of the measurement. From Figure 10, it can be seen that
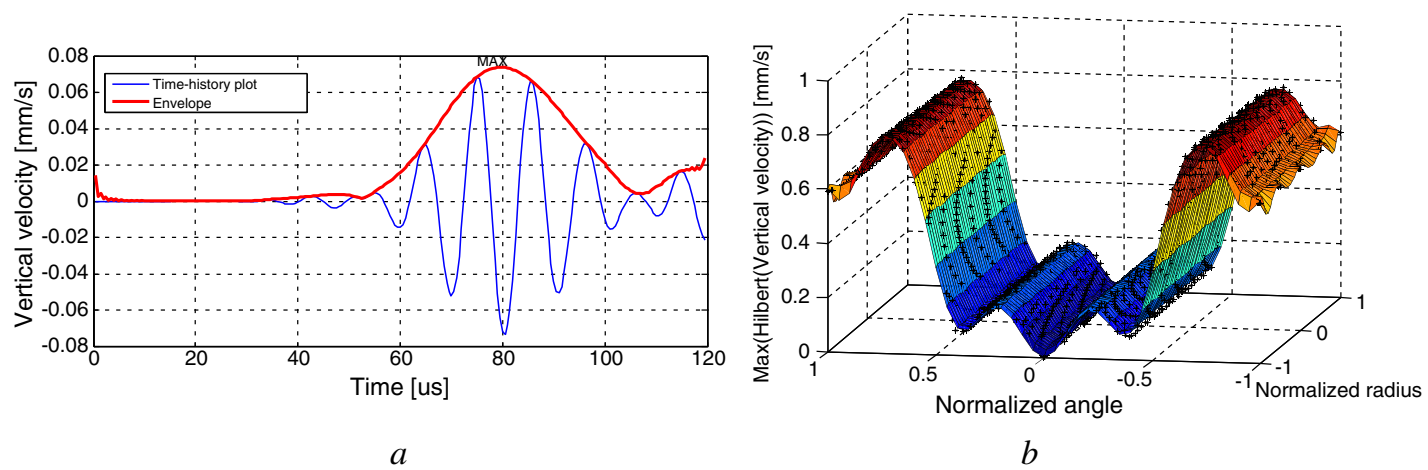

Figure 9. Example of the beampattern calculation: (a) snapshot of the vertical velocity for a node and (b) extremes of the envelopes. 


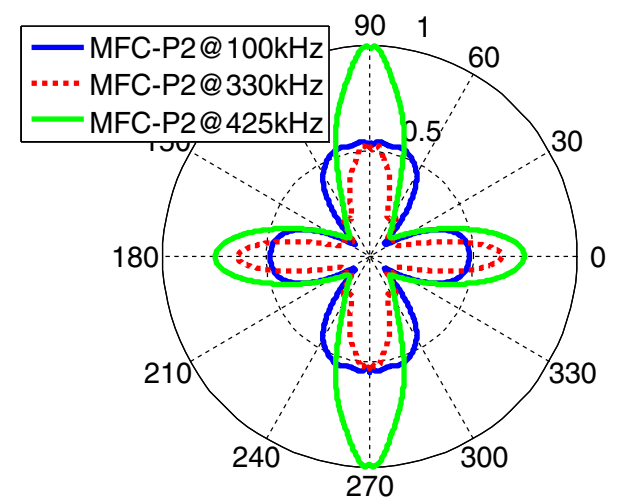

Figure 10. Normalized beampatterns of MFC-M2814-P2 at excitation frequencies 100, 330, and $425 \mathrm{kHz}$.

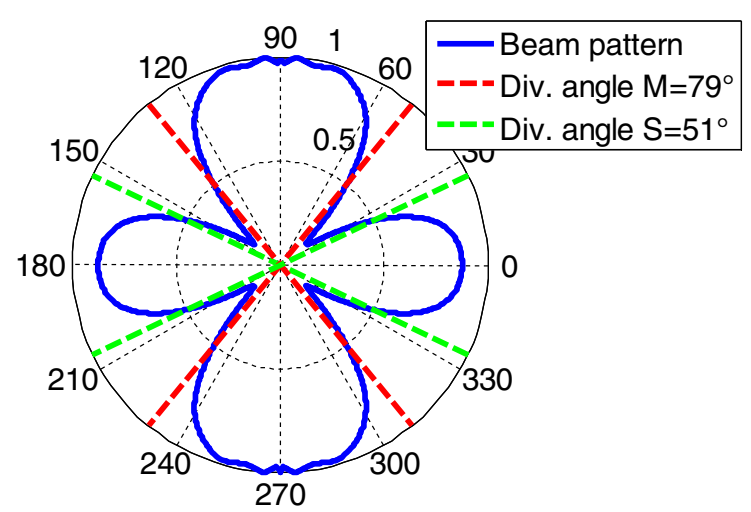

Figure 11. Normalized beampattern of the MFC-M2814-P2 at $100 \mathrm{kHz}$.

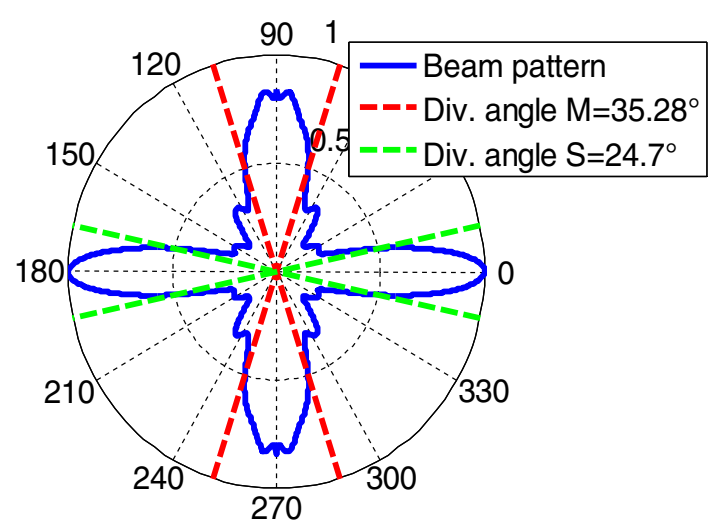

Figure 12. Normalized beampattern of the MFC-M2814-P2 at $330 \mathrm{kHz}$.

the highest amplitude of the propagating wave occurs for frequency $425 \mathrm{kHz}$. For this frequency, the main-to-side-lobe amplitude ratio (MSLR) is 1.3 ; for lower frequencies, 330 and $100 \mathrm{kHz}$, this ratio decreased to 0.84 and 1.1 , respectively.

\subsection{Numerical results for the MFC-P1 with d33 effect}

The second simulation series was performed for the MFC-M2814-P1 transducer, which exhibits d33 piezoelectric effect. The highest amplitude of the generated wave was observed again for excitation frequency $425 \mathrm{kHz}$, as can be seen in Figure 14. The main lobe width for that frequency was $31.6^{\circ}$ 


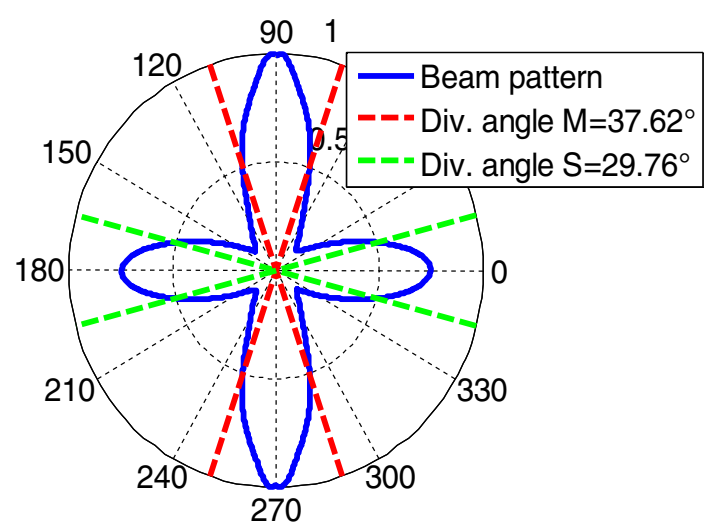

Figure 13. Normalized beampattern of the MFC-M2814-P2 at $425 \mathrm{kHz}$.

(Figure 15). The amplitudes of the waves generated for lower frequencies were approximately two times lower than those for $425 \mathrm{kHz}$, and the beampatterns had much wider main lobes $\left(72^{\circ}\right.$ for $100 \mathrm{kHz}$; Figures 16 and 17). It appears that the MSLR also depends on the excitation frequency: for $100 \mathrm{kHz}$, this ratio was 10.8; and for the higher frequencies, 330 and $425 \mathrm{kHz}$, it was reduced to 6.5 and 7.3 , respectively.

\subsection{Numerical results for double-sided interdigital transducer with d31 effect}

Comparison of the simulation results for the IDT-DS4 presented in Figure 18 shows that the best performance was obtained for the nominal excitation frequency equal to $330 \mathrm{kHz}$. The amplitude at this

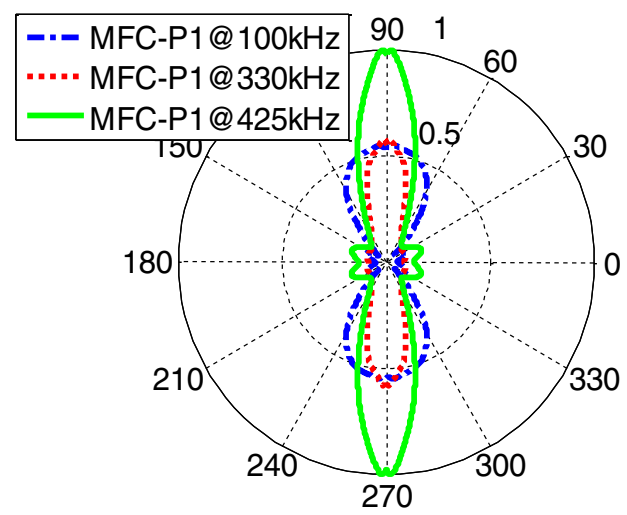

Figure 14. Normalized beampatterns of the MFC-M2814-P1 at excitation frequencies 100, 330, and $425 \mathrm{kHz}$.

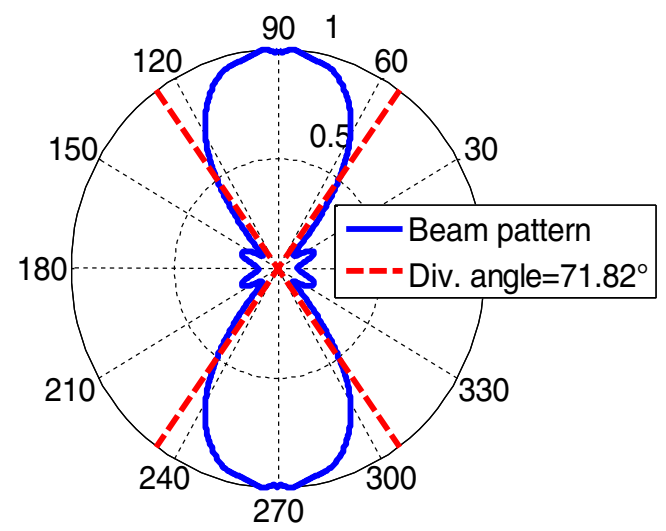

Figure 15. Normalized beampattern of the MFC-M2814-P1 at $100 \mathrm{kHz}$. 


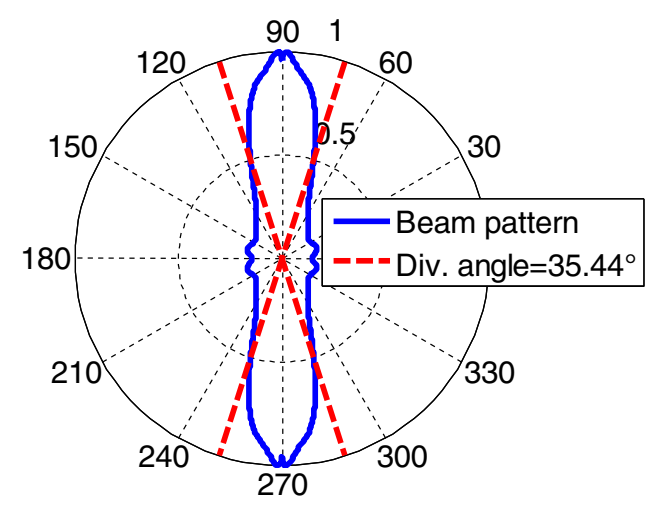

Figure 16. Normalized beampattern of the MFC-M2814-P1 at $330 \mathrm{kHz}$.

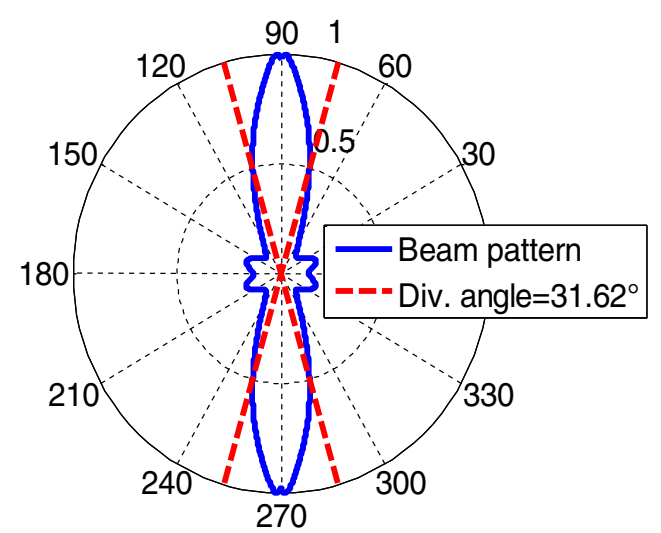

Figure 17. Normalized beampattern of the MFC-M2814-P1 at $425 \mathrm{kHz}$.

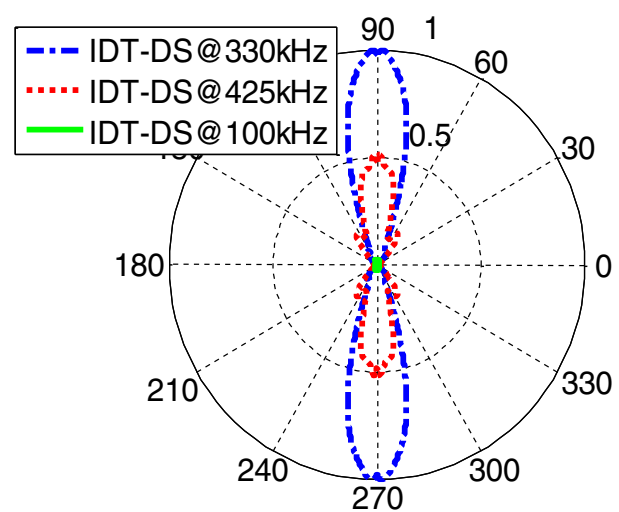

Figure 18. Normalized beampatterns of the IDT-DS4 at excitation frequencies 100, 330, and $425 \mathrm{kHz}$.

frequency is almost two times higher than that obtained at $425 \mathrm{kHz}$ and over 35 times higher than at $100 \mathrm{kHz}$ From Figure 19, it can be seen that the main lobes are characterized by very good MSLR and narrow divergence angle equal to $30^{\circ}$. For higher excitation frequencies, the beampattern, presented in Figure 20, remains similar, but well-pronounced side lobes appear at approximately $30^{\circ}$ on both sides of the main lobe. For excitation frequency $100 \mathrm{kHz}$, the simulated beampattern, shown in Figure 21, takes the form of a butterfly with wide main lobes (divergence angle up to $86^{\circ}$ ) and considerable side lobes. 


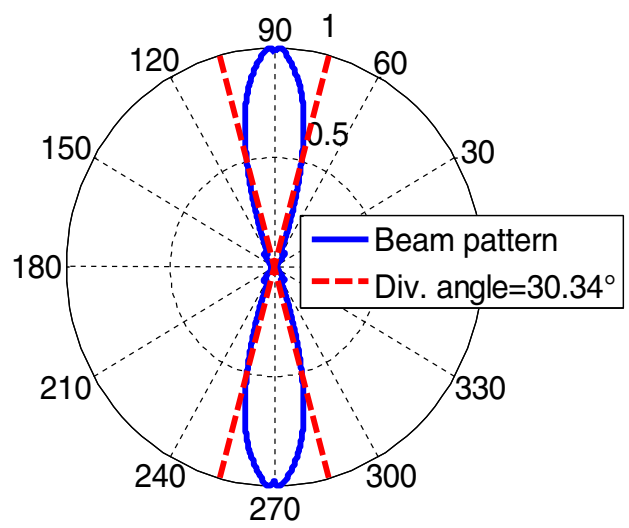

Figure 19. Normalized beampattern of the IDT-DS4 at $330 \mathrm{kHz}$.

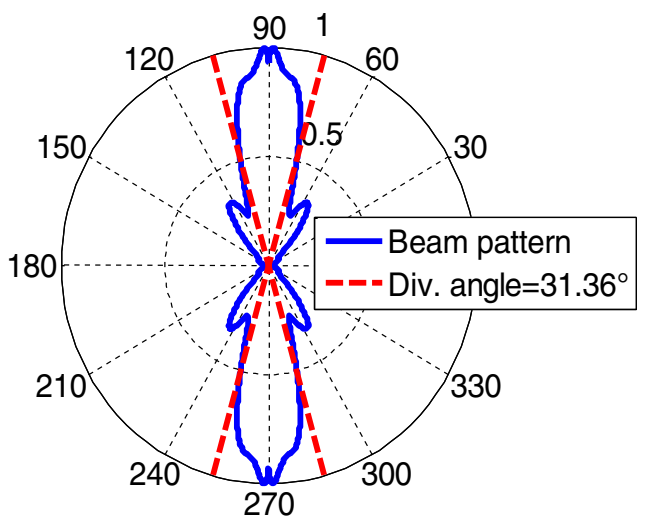

Figure 20. Normalized beampattern of the IDT-DS4 at $425 \mathrm{kHz}$.

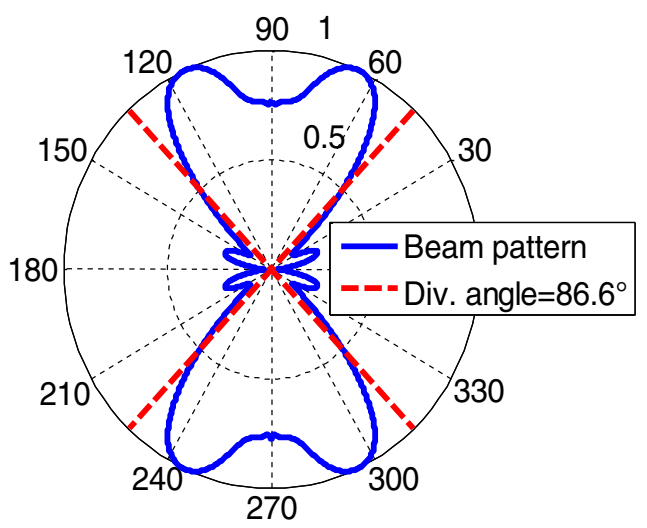

Figure 21. Normalized beampattern of the IDT-DS4 at $100 \mathrm{kHz}$.

The best MSLR of the IDT-DS4 equal 110 is observed at its nominal frequency $330 \mathrm{kHz}$; for other frequencies, it is much lower but still higher than that for the MFC-M2814-P1 transducer: 28 for $100 \mathrm{kHz}$ and 39 for $425 \mathrm{kHz}$, respectively.

\subsection{Numerical results: discussion}

Results obtained during the simulation with MFC-2814-P2 showed that the transducer is not able to generate directional wave (Figure 10). During investigation, an additional series of simulations were 
carried with the model of the PZT stack transducer, whose dimensions were the same as the MFC transducers. Results obtained during these simulations are presented in Figure 22.

It can be seen that in both cases (the MFC and PZT), the transducers generate waves in all four directions simultaneously, with the main radiation direction and amplitudes changing with the excitation frequency.

Figure 23 provides comparison between the best results from each type of the investigated transducers. It was defined that the best results should have the highest amplitude related to the highest MSLR. Because only a single result was chosen from each of the tests, the results for the following excitation frequencies were also considered: $425 \mathrm{kHz}$ for the MFC-P1/P2 and $330 \mathrm{kHz}$ for the IDT-DS4.

It is visible at a glance that the beampattern generated by the IDT-DS4 transducer has not only the highest MSLR but also the highest main lobe amplitude and the smallest main lobe width.

The numerical results confirm the conclusions from the theoretical analysis that finger separation matched to the length of the generated wave amplifies the amplitude of the generated wave for a given frequency. In all tested frequencies, the calculated divergence angles are similar, but higher amplitude obtained for the IDT-DS4 leads to better MSLR.

\section{EXPERIMENTS}

\subsection{Experimental setup}

The transducers investigated in the experiments were mounted on the $1000 \times 1000 \times 4 \mathrm{~mm}$ aluminum plate by using Loctite 3430 epoxy bond (Henkel AG \& Co. KGaA Henkelstraße 67, Düsseldorf, Germany) that was cured before the tests for at least $24 \mathrm{~h}$. The excitation signals were generated by

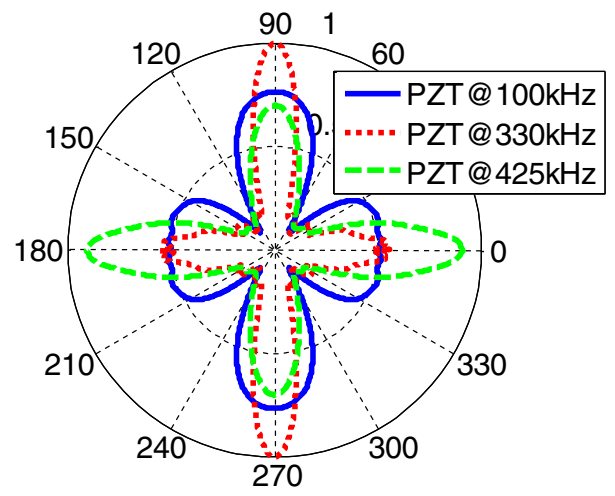

Figure 22. Normalized beampatterns of PZT stack transducer at excitation frequencies 100, 330 and $425 \mathrm{kHz}$.
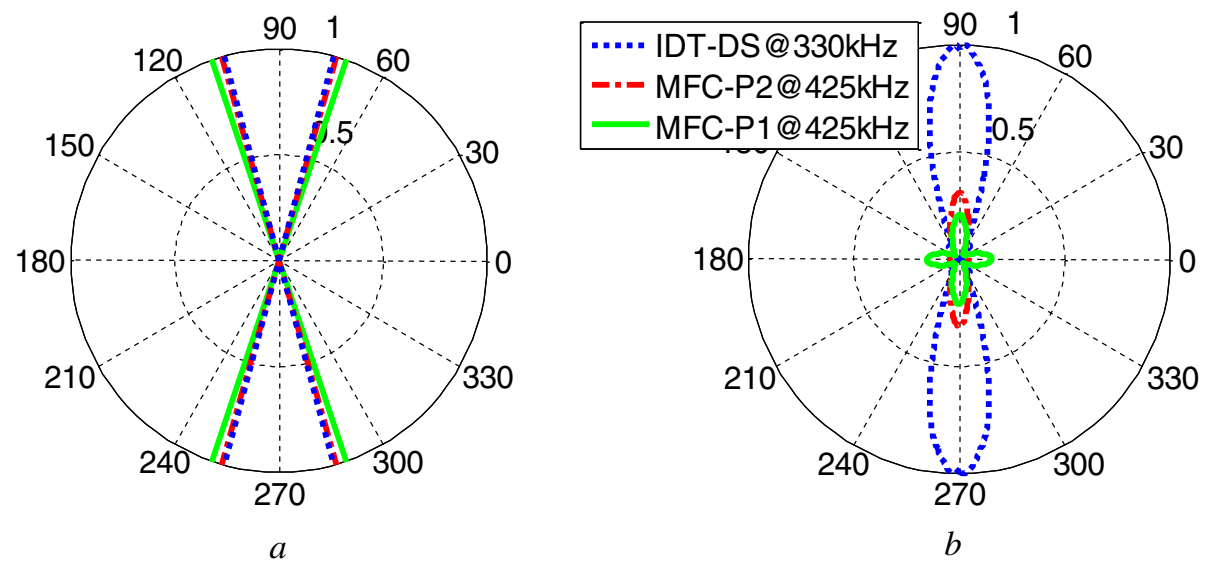

Figure 23. Comparison of the (a) normalized simulated divergence angles and (b) beampatterns of the investigated transducers. 
the specially designed for SHM applications Piezo-Acquisition System PAS-8000 and PAQ-16000D from EC-Electronics, Poland. The measurements of out-of-plane vibrations were performed using the Polytec PSV-400 scanning laser vibrometer (Polytec GmbH Polytec-Platz 1-7, Waldbronn, Germany) located in front of the tested plate as shown in Figure 24. The vibrometer consists of two main parts: the PSC-I-400 sensor head and the OFV-5000 vibrometer controller.

To improve the reflective properties of the surface, the measured area was covered with the retroreflecting material dedicated for laser vibrometry tests. Sampling frequency of the vibrometer was set at $5.12 \mathrm{MHz}$, and the sensitivity at $20 \mathrm{~mm} / \mathrm{s} / \mathrm{V}$. To suppress the influence of the noise, the measurements were repeated four times in each point and subsequently averaged and filtered with bandpass filter with bandwidth $100 \mathrm{kHz}$ ( $\pm 50 \mathrm{kHz}$ round the excitation frequency). The excitation signals used in the experiment consisted of five-cycle tone sine burst modulated with Hanning window. The burst frequencies used in the experiments were the same as in the FEM simulations: 100, 330, and $425 \mathrm{kHz}$.

The transducers' beampatterns were calculated for each of the excitation frequencies on the basis of the vibrometer measurements (Figure 25). For each of the measured points, the Hilbert transform was

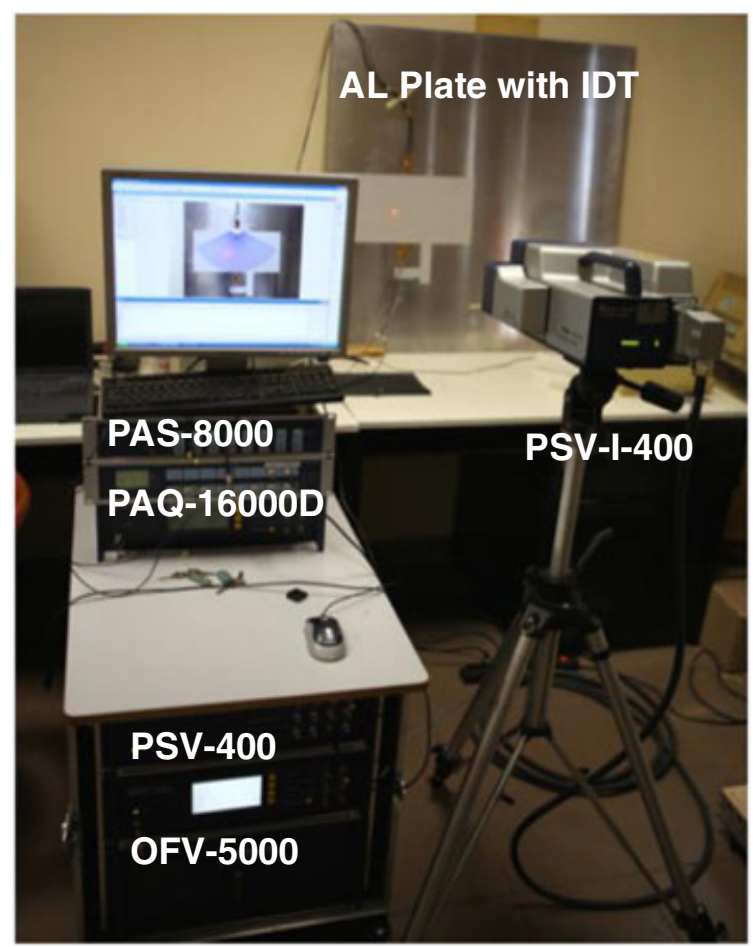

Figure 24. Experimental setup used to test designed transducers.

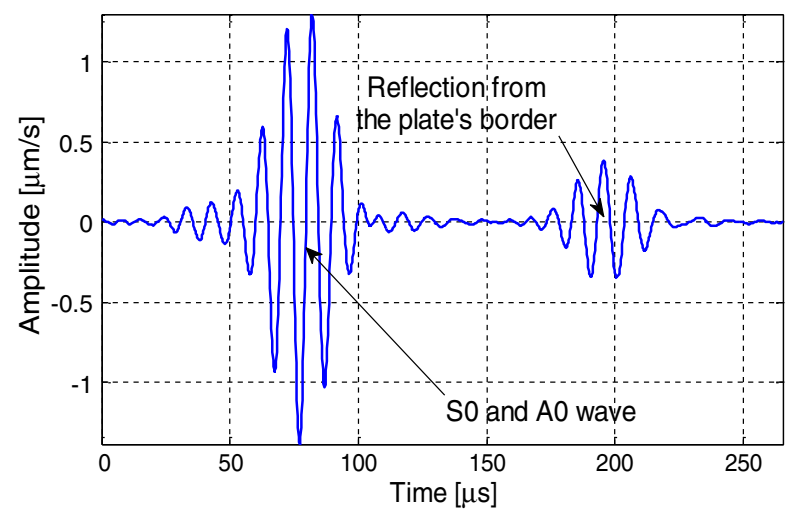

Figure 25. Example of Lamb wave propagation in 4-mm-thick aluminum plate. 
used to determine envelopes of the snapshots of the out-of-plane velocity. Next, the maximal value of each envelope was found and used for the beampattern calculation. Finally, the calculated beampatterns were normalized and smoothed by a moving average filter. In the following discussions, the beampatterns obtained from the vibrometer data are presented and compared with the simulated ones. Example snapshots registered at the transducer axis at the distance $150 \mathrm{~mm}$ from the transducers are also shown.

\subsection{Experimental results for the MFC-P2 with d31 effect}

Results obtained during the investigation of the transducer MFC-M2814-P2 are presented in Figures 26 and 27.

Comparison of the beampatterns presented in Figure 26 shows that the MFC-M2814-P2 generates both front and side lobes for all three test frequencies. The front lobes have similar amplitudes for all three frequencies, whereas the side lobes' amplitude varies with frequency and achieves its largest value for $330 \mathrm{kHz}$. The front lobe width is largest for the lowest frequency, $100 \mathrm{kHz}$, and it decreases for the higher frequencies. Variations in the main radiation direction pronounced in the experimental data presented in Figure 26 are similar to those observed by Callet et al. [29]. At excitation frequency $100 \mathrm{kHz}$, the MSLR is 1.06 , whereas for higher frequencies, this ratio is reduced to 0.4 for $330 \mathrm{kHz}$ and 0.6 for $425 \mathrm{kHz}$. Experiments show that relatively high mode selectivity was achieved only for the lowest frequency, $100 \mathrm{kHz}$, where only a single-mode wave was observed; for higher frequencies, additional modes appear as it can be seen in Figure 27.

Diagrams provided in Figure 28(a-c) enable comparison of the simulation and experimental data for the MFC-M2814-P2. Note that the maximal experimentally obtained amplitudes, given in the lower-right corner of each diagram, correspond to the radius of the largest circle. Those amplitudes were used for

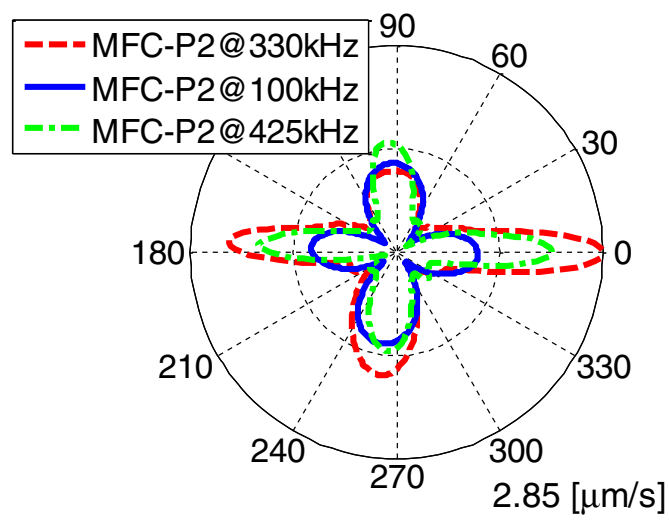

Figure 26. Normalized beampatterns of the MFC-M2814-P2 at excitation frequencies 100, 330, and $425 \mathrm{kHz}$.

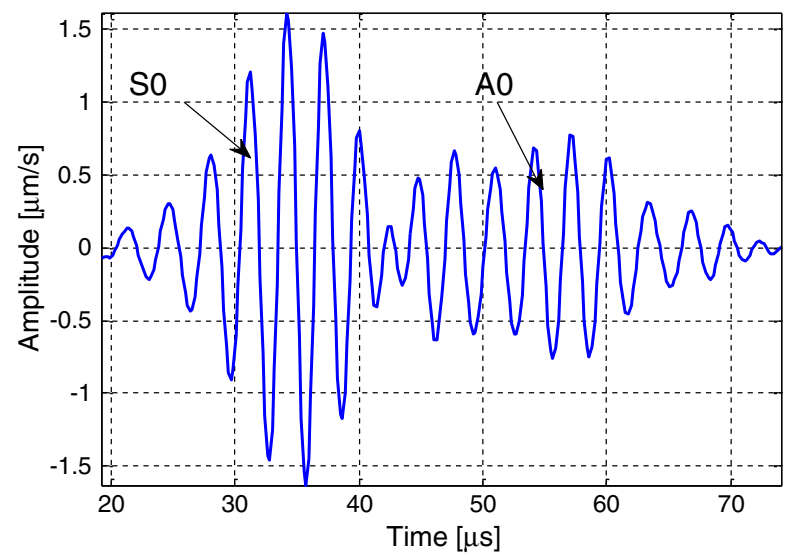

Figure 27. Snapshot of the signal measured for the MFC-M2814-P2 at excitation frequency $330 \mathrm{kHz}$. 


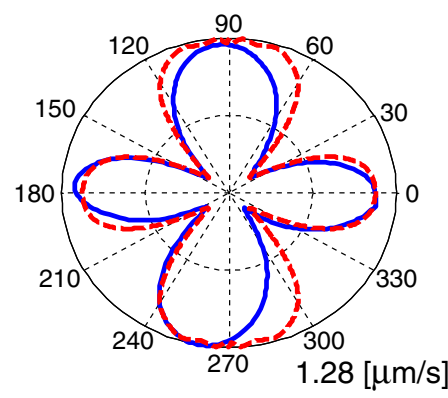

a)

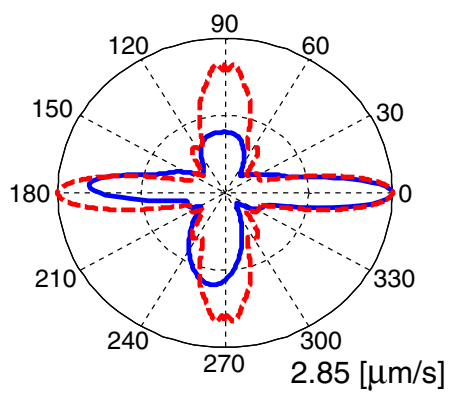

b)

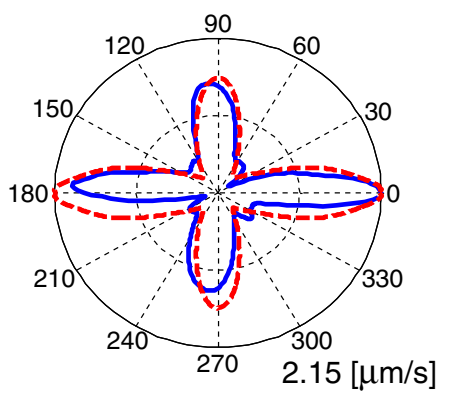

c)

Figure 28. Normalized simulated (dashed) and experimental (solid) beampatterns of the MFC-M2814-P2 for different frequencies: (a) $100 \mathrm{kHz}$, (b) $330 \mathrm{kHz}$, and (c) $425 \mathrm{kHz}$. Scale in micrometer per second.

the amplitude normalization of the respective simulated beampatterns. Figure $28(\mathrm{a}-\mathrm{c})$ demonstrates good agreement between the simulations and experiments in terms of the shape of the generated beampatterns.

\subsection{Experimental results for the MFC-P1 with d33 effect}

Normalized results of the experiments that were performed with the MFC-M2814-P1 can be compared in Figure 29. In the figure, it can be seen that this transducer generated a bidirectional wave in the directions perpendicular to the electrodes for all of the tested frequencies.

Similarly to the MFC-M2814-P2, in this case, better mode selectivity was observed at the lowest frequency $(100 \mathrm{kHz})$ where only a single mode could be distinguished with the frequency corresponding to the excitation. With the change of the excitation frequency, additional modes of GWs were generated, which can be seen in Figure 30.

Contrary to the previous transducer, the shape of the generated beampattern (Figure 29) changed with the excitation frequency. For higher frequencies, the bidirectional character of the wave was preserved; however, additional side lobes appeared with amplitudes and localizations, depending on the frequency. In this case, the divergence angle could be estimated only for excitation frequency $100 \mathrm{kHz}$, and it was equal to $55^{\circ}$ and the MSLR value was 4.1 .

Similarly to the previous transducer, in this case, also a good agreement between the experimental and simulation data can be observed, especially at the lowest excitation frequency, in Figure 31(a); for higher frequencies, additional side lobes, which were not present in the numerical data, are visible in the experimental results. Note, however, that the velocities corresponding to the beampattern amplitudes, shown in Figure 31, were approximately one order of magnitude lower that those measured for the MFC-M2814-P2.

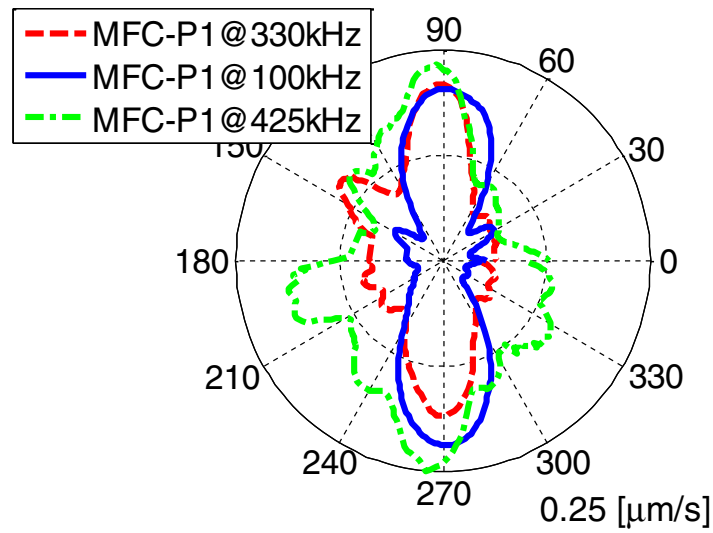

Figure 29. Normalized beampatterns of the MFC-M2814-P1 at excitation frequencies 100, 330, and $425 \mathrm{kHz}$. 


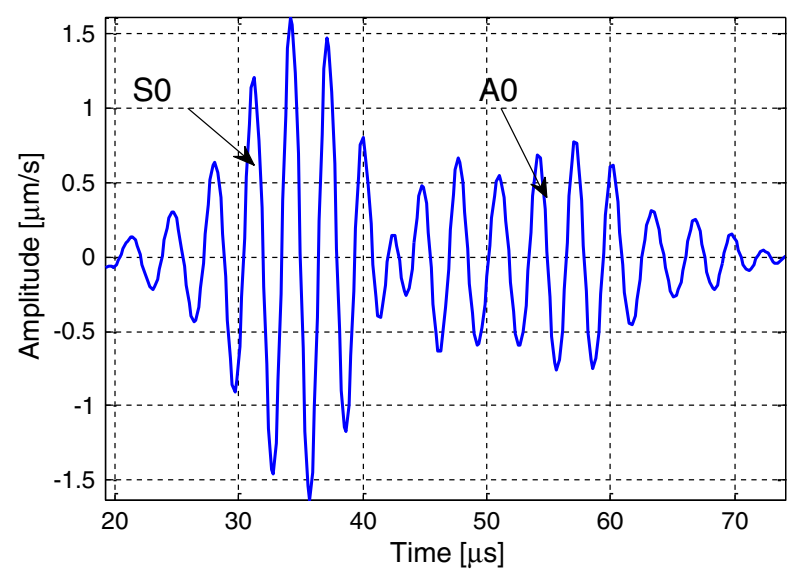

Figure 30. Snapshot of the measured signal at excitation frequency $330 \mathrm{kHz}$ for the MFC-M2814-P1.

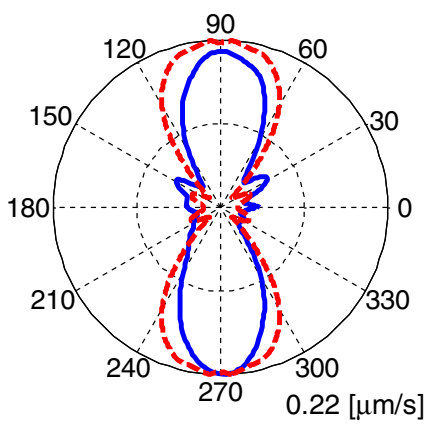

a)

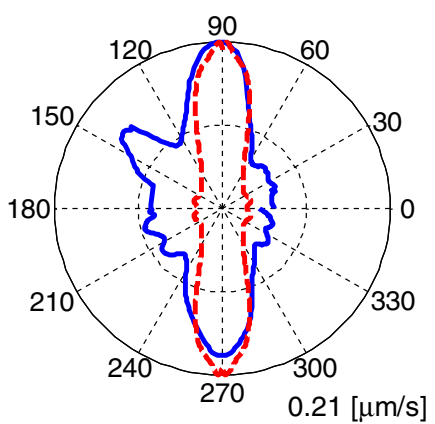

b)

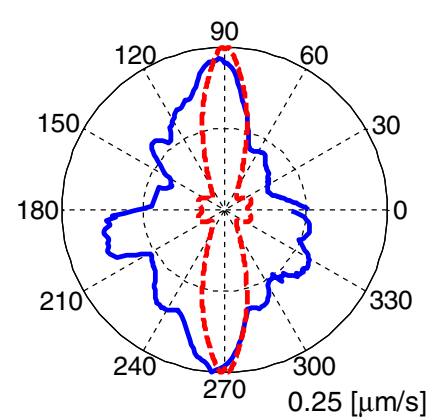

c)

Figure 31. Normalized simulated (dashed) and experimental (solid) beampatterns of the MFC-M2814-P1 for different frequencies: (a) $100 \mathrm{kHz}$, (b) $330 \mathrm{kHz}$, and (c) $425 \mathrm{kHz}$. Scale in micrometer per second.

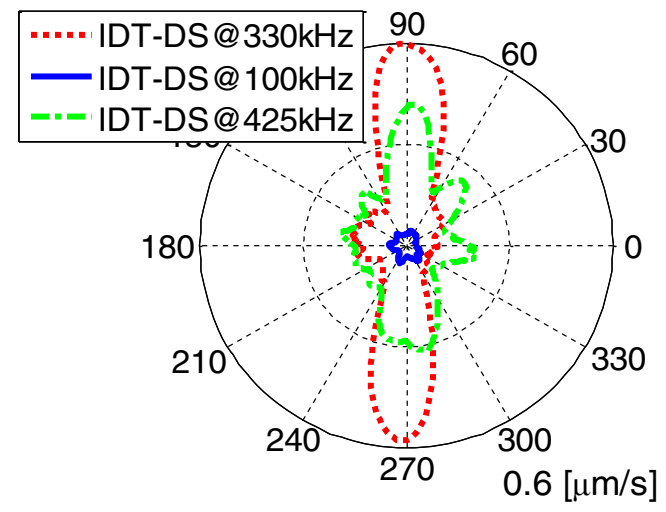

Figure 32. Normalized beampatterns of the IDT-DS4 at excitation frequencies 100, 330, and $425 \mathrm{kHz}$.

\subsection{Experimental results for the double-sided interdigital transducer with d31 effect}

The third investigated transducer was the custom-made IDT-DS with the $\mathrm{d} 31$ piezoelectric effect made of the same substrate as the MFC-M2814-P2. The IDT-DS4 was designed to work at a particular nominal frequency $(330 \mathrm{kHz})$, but for comparison with the MFCs, it was excited with the same frequencies as the transducers presented earlier. The beampattern characteristics of this transducer, shown in Figure 32, demonstrate its ability to generate a bidirectional wave in the direction perpendicular to its comb electrodes. 
Similarly to the simulation results, the best performance of the transducer was observed at frequency $330 \mathrm{kHz}$. Time and frequency domain analysis of the results shows that three modes were generated at excitation frequency $330 \mathrm{kHz}$ : the A0 and S0 modes at frequency $330 \mathrm{kHz}$ and a weak S0 mode at $425 \mathrm{kHz}$, which was the symmetric mode corresponding to the electrodes spacing as shown in Figure 5.

This mode was excited by the small amount of energy present in the higher frequency band of the $330 \mathrm{kHz}$ burst. The S0 modes propagate with higher speed $(4.8$ and $4.1 \mathrm{~km} / \mathrm{s}$, respectively) than the A0, which has a velocity of $3.1 \mathrm{~km} / \mathrm{s}$ (Table B1). The calculated MSLR at frequency $330 \mathrm{kHz}$ was 4.6 , and the divergence angle was equal to $34^{\circ}$ (Figure 32).

The result obtained at frequency $100 \mathrm{kHz}$ is very difficult to interpret because the amplitude of the generated wave is very low. From Figure 33(a), it can be seen that the maximal amplitude for $100 \mathrm{kHz}$ is 10 times lower than that for $330 \mathrm{kHz}$, and the beampattern does not indicate any well-pronounced directionality. At the highest frequency, the main lobe is better pronounced, but the main lobe amplitude is lower than at frequency $330 \mathrm{kHz}$, and additional side lobes are also visible. Also at this frequency, besides the modes related to the excitation frequency, the slow mode related to the electrode spacing can be distinguished (A0 at $330 \mathrm{kHz}$ ).

Comparison of the simulation and experimental results for the IDT-DS4, presented in Figure 34(a-c), shows very good agreement between simulations and experiments for the nominal frequency. Also for frequency $425 \mathrm{kHz}$, the beampatterns are similar including the presence of additional side lobes generated at the edges of the transducer.

\section{DISCUSSIONS}

Experimental results have shown that properties of all considered transducers vary with the change of excitation frequency. For further discussion, only those frequencies where the highest MSLR and the

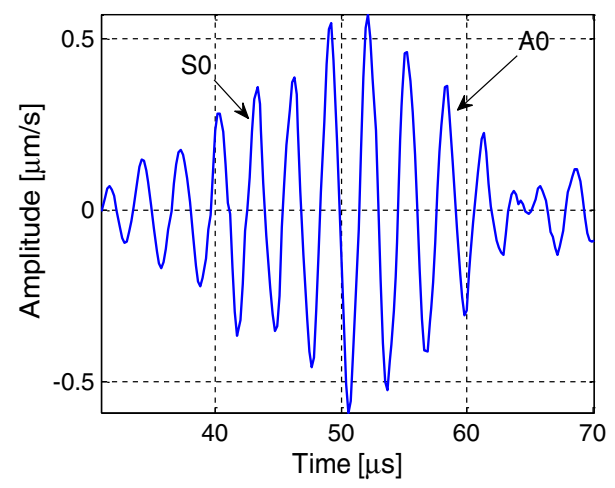

Figure 33. Snapshot of the signal measured for the IDT-DS4 at excitation frequency $330 \mathrm{kHz}$.

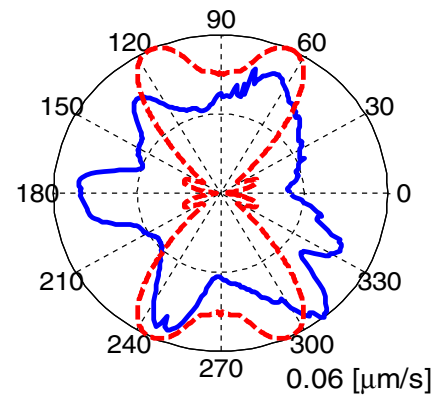

a)

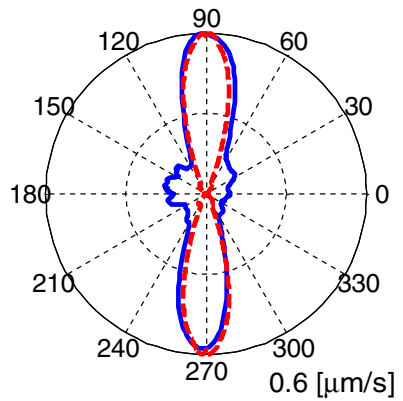

b)

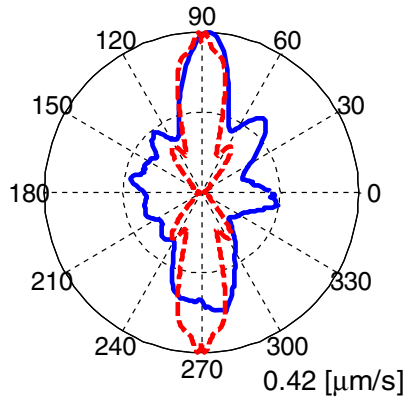

c)

Figure 34. Normalized simulated (dashed) and experimental (solid) beampatterns of the IDT-DS4 for different frequencies: (a) $100 \mathrm{kHz}$, (b) $330 \mathrm{kHz}$, and (c) $425 \mathrm{kHz}$. Scale in micrometer per second. 
best mode selectivity could be observed have been chosen. In the results presented in this paper, such situation occurs at low frequencies $(100 \mathrm{kHz})$ for the MFC-M2814-P1/P2 transducers and at frequency $330 \mathrm{kHz}$ for the IDT-DS4. These are compared in Figure 35, providing the beampatterns with the highest MSLR generated by the investigated transducers during experiments, with a scale factor for the amplitudes equal $1.28 \mu \mathrm{m} / \mathrm{s}$.

In Figure 35, it can be seen that the best directionality in terms of side lobe level, among the investigated transducers, was achieved by the IDT-DS4, which had the MSLR approximately 5, whereas the MFC-M2814-P1 had approximately 4, and the MFC-M2814-P2 had only 1. Also, the best directionality in terms of the divergence angle was observed for the IDT-DS4 that had the main lobe width of $34^{\circ}$. This can be compared with $55^{\circ}$ for the MFC-M2814-P1 and $67^{\circ}\left(50^{\circ}\right.$ side wave) for the MFC-M2814-P2.

On the basis of the performed tests, only a qualitative comparison may be carried out because only a single transducer of each type was tested. The transducers were bonded manually in different positions on the plate, which may have lead to differences in bond quality between the transducers and the plate. To reduce the negative influence of these factors, multiple tests should be performed, and then on the basis of statistical analysis, a quantitative comparison could be performed.

Experimental results confirm the numerical results in terms of the beampatterns shape; however, contrary to the numerical results, the highest amplitude among the tested transducers was generated by the MFC-M2814-P2. In the experiments, it was two times higher than that generated by the IDT-DS4 and over six times higher than the amplitude of the MFC-M2814-P1. The source of this difference may be any of the factors mentioned earlier (bonding quality and material properties of the plate or of the piezocomposite) as well as imperfections of the numerical model.

The major differences observed between the numerical and experimental results are the differences in the amplitude level at different frequencies, which is especially visible for IDT-DS4. To identify the influences of selected parameter that may be the source of those, the influence of the bonding layer on the generated wave was investigated using FE simulations.

An additional layer of glue ( $50 \mu \mathrm{m}$ thick) was added between the IDT-DS4 and the aluminum plate, and for such model, the simulations for all three frequencies were repeated. Results obtained during these simulations are compared with the original results in Figure 36.

It is visible that for low frequencies, the bonding layer significantly damps generated waves (Figure 36(a)). However, for higher frequencies, the opposite effect is observed: the generated signals have higher amplitudes than the ones observed without the presence of bonding layer. Those changes also affect the amplitude-frequency characteristics of the transducer, as presented in Figure 37.

If the beampatterns shown in Figure 37 are compared with those obtained for the model without bonding layer (Figure 18), it can be seen that the difference between the amplitudes of the signal generated at 330 and $425 \mathrm{kHz}$ is minimal. However, the shapes and the calculated divergence angles for all of the studied frequencies remain the same.

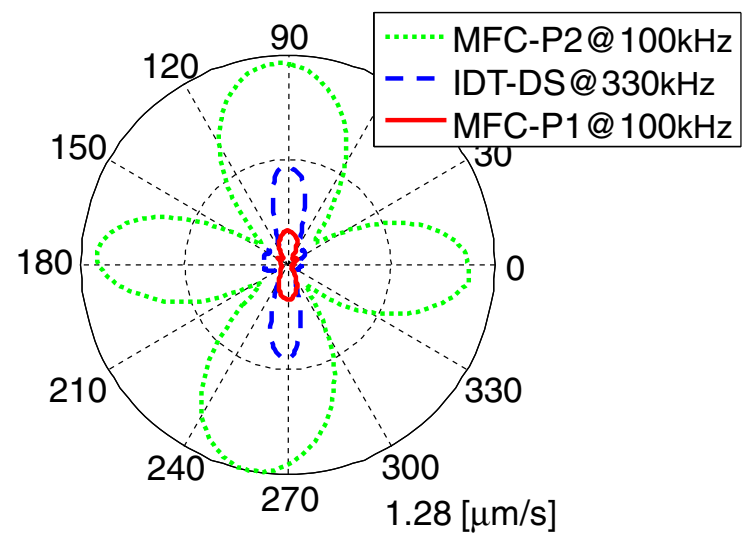

Figure 35. Experimental beampatterns of the investigated transducers. 


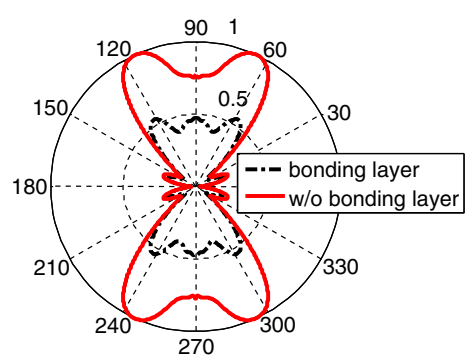

a)

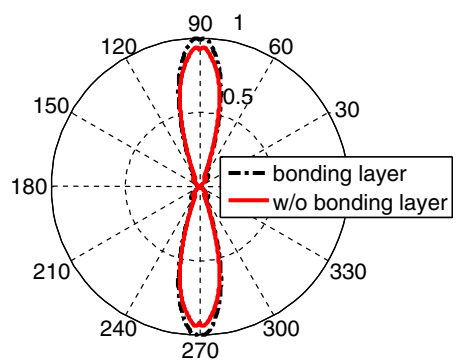

b)

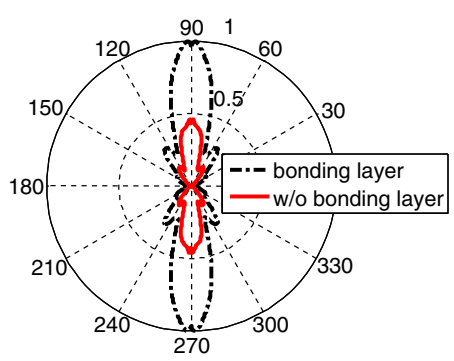

c)

Figure 36. Normalized simulated beampatterns of the IDT-DS4 for different frequencies with (solid) and without (dashed) bonding layer: (a) $100 \mathrm{kHz}$, (b) $330 \mathrm{kHz}$, and (c) $425 \mathrm{kHz}$.

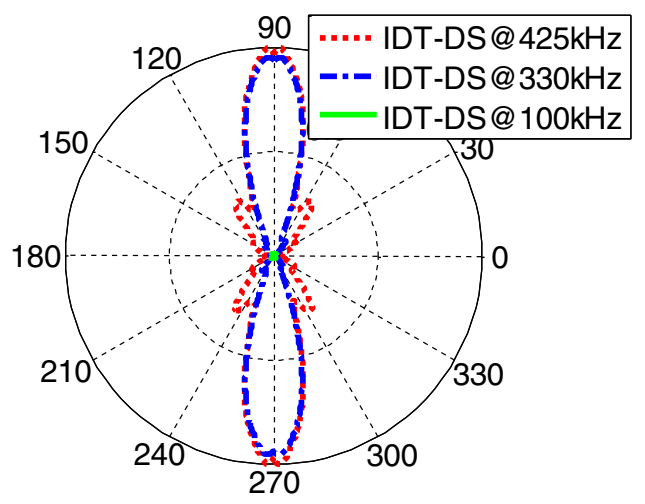

Figure 37. Normalized beampatterns of the IDT-DS4 at excitation frequencies 100, 330, and $425 \mathrm{kHz}$ when bonding layer is included in the model.

A series of numerical and experimental tests are currently performed by the authors with the aim of identifying and explaining the influence of the bonding layer as well as other factors on the properties of LWs generated by the IDT.

\section{CONCLUSIONS}

In this paper, two transducer types built using the MFC substrate with different electrodes, that is, the standard dense electrodes and the IDT-DS electrodes, were compared using numerical simulations and experiments. Both types of the investigated transducers were in the form of thin and flexible patches suitable for SHM applications. Two types of the MFC substrates were used for the transducers with dense electrodes, the d33 polarized for MFC-P1 and the d31 for MFC-P2, whereas the d31 MFC was used for the IDT-DS transducer.

The results of numerical simulations and experiments presented in this paper showed the superiority of the IDT transducer in comparison with the other two types, which, for its nominal frequency, has the narrowest main lobe linked with relatively high amplitude and has the lowest level of side lobes. Moreover, the IDT-DS showed mode selectivity related to the distance between its electrodes matched to the wavelength of a particular wave mode (A0 at $330 \mathrm{kHz}$ ).

This means that the MFC-based IDT-DS is a better choice for SHM applications than the MFC-P1 and MFC-P2 with dense electrodes. High amplitude and the possibility of tuning up operating frequency to the frequency where dispersion of the excited mode is minimal make the IDT very suitable for long-range ultrasonic testing. Until now, the IDT transducers have been manufactured using the PZT ceramics or PVDF as piezoelectric layer, which results in brittle (PZT) or weak (PVDF) transducers. The authors have proven that the IDT-DS concept, presented in this paper, unifies the advantages of the IDT and MFC because it enables manufacturing flexible transducers capable of generating high-amplitude waves characterized by high directionality. 
Appendix A: Piezoelectric properties of composites used in simulations.

Table A1. Piezoelectric properties of d31-type composite used in IDT-DS4 and MFC-M2814-P2.

\begin{tabular}{|c|c|c|}
\hline $\begin{array}{l}c^{E}{ }_{11}=3.94 \times 10^{10} \mathrm{~Pa} \\
c^{E}{ }_{12}=1.29 \times 10^{10} \mathrm{~Pa} \\
c^{E}{ }_{44}=0.55 \times 10^{10} \mathrm{~Pa} \\
e_{31}=-7.12 \mathrm{C} / \mathrm{m}^{2} \\
e_{24}=-17.03 \mathrm{C} / \mathrm{m}^{2} \\
\varepsilon^{\varepsilon}{ }_{11}=237.2 \varepsilon_{0} \mathrm{C} / \mathrm{V} / \mathrm{m}\end{array}$ & $\begin{aligned} c_{22}^{E} & =2.03 \times 10^{10} \mathrm{~Pa} \\
c^{E}{ }_{13} & =0.83 \times 10^{10} \mathrm{~Pa} \\
c^{E_{55}} & =0.55 \times 10^{10} \mathrm{~Pa} \\
e_{33} & =12.1 \mathrm{C} / \mathrm{m}^{2} \\
e_{15} & =-17.03 \mathrm{C} / \mathrm{m}^{2} \\
\varepsilon_{22}^{\varepsilon} & =237.2 \varepsilon_{0} \mathrm{C} / \mathrm{V} / \mathrm{m}\end{aligned}$ & $\begin{aligned} c^{E}{ }_{33} & =3.25 \times 10^{10} \mathrm{~Pa} \\
c^{E_{23}^{23}} & =0.53 \times 10^{10} \mathrm{~Pa} \\
c^{E_{66}} & =1.31 \times 10^{10} \mathrm{~Pa} \\
e_{32} & =-4.53 \mathrm{C} / \mathrm{m}^{2} \\
\rho & =7000 \mathrm{~kg} / \mathrm{m}^{3} \\
\varepsilon_{33}^{\varepsilon} & =143.4 \varepsilon_{o} \mathrm{C} / \mathrm{V} / \mathrm{m}\end{aligned}$ \\
\hline
\end{tabular}

Table A2. Piezoelectric properties of d33-type composite used in MFC-M2814-P1.

\begin{tabular}{lcc}
\hline$c^{E}{ }_{11}=3.94 \times 10^{10} \mathrm{~Pa}$ & $c^{E}{ }_{22}=2.03 \times 10^{10} \mathrm{~Pa}$ & $c^{E}{ }_{33}=3.25 \times 10^{10} \mathrm{~Pa}$ \\
$c^{E}{ }_{12}=1.29 \times 10^{10} \mathrm{~Pa}$ & $c^{E_{13}}=0.83 \times 10^{10} \mathrm{~Pa}$ & $c^{E_{23}}=0.53 \times 10^{10} \mathrm{~Pa}$ \\
$c^{E}{ }_{44}=0.55 \times 10^{10} \mathrm{~Pa}$ & $c^{E_{55}}=0.55 \times 10^{10} \mathrm{~Pa}$ & $c^{E_{66}}=1.31 \times 10^{10} \mathrm{~Pa}$ \\
$e_{31}=13.62 \mathrm{C} / \mathrm{m}^{2}$ & $e_{33}=-4.1 \mathrm{C} / \mathrm{m}^{2}$ & $e_{32}=0.55 \mathrm{C} / \mathrm{m}^{2}$ \\
$e_{24}=-17.03 \mathrm{C} / \mathrm{m}^{2}$ & $e_{15}=-17.03 \mathrm{C} / \mathrm{m}^{2}$ & $\rho=7000 \mathrm{~kg} / \mathrm{m}^{3}$ \\
$\varepsilon^{\varepsilon}=141.2 \varepsilon_{0} \mathrm{C} / \mathrm{V} / \mathrm{m}$ & $\varepsilon^{\varepsilon}{ }_{22}=141.2 \varepsilon_{0} \mathrm{C} / \mathrm{V} / \mathrm{m}$ & $\varepsilon_{33}^{\varepsilon}=141.2 \varepsilon_{0} \mathrm{C} / \mathrm{V} / \mathrm{m}$ \\
\hline
\end{tabular}

Appendix B: Group velocities and respected Time Of Flight (TOF) of the A0, S0, and A1 modes.

Table B1. Group velocities and respected TOF of the A0, S0, and A1 modes at tested frequencies corresponding to the dispersion plots in Figure 5.

\begin{tabular}{|c|c|c|c|c|c|c|}
\hline \multirow[b]{2}{*}{ Mode } & \multicolumn{2}{|c|}{$100 \mathrm{kHz}$} & \multicolumn{2}{|c|}{$330 \mathrm{kHz}$} & \multicolumn{2}{|c|}{$425 \mathrm{kHz}$} \\
\hline & Velocity $(\mathrm{km} / \mathrm{s})$ & TOF $(\mu s)$ & Velocity $(\mathrm{km} / \mathrm{s})$ & $\mathrm{TOF}(\mu \mathrm{s})$ & Velocity $(\mathrm{km} / \mathrm{s})$ & TOF $(\mu \mathrm{s})$ \\
\hline So & 5.3 & 28.2 & 4.8 & 31.4 & 4.1 & 37.0 \\
\hline A0 & 2.8 & 54.2 & 3.1 & 47.9 & 3.1 & 48.0 \\
\hline A1 & - & - & - & - & 2.3 & 66.1 \\
\hline
\end{tabular}

\section{ACKNOWLEDGEMENTS}

The work presented in this paper has been financed by the program MISTRZ from the Foundation for Polish Science, which is greatly acknowledged.

\section{REFERENCES}

1. Raghavan A, Cesnik CES. Review of guided-wave structural health monitoring. The Shock and Vibration Digest 2007; 39(2):91-114.

2. Roy S, Deanna RG, Izad A, Mehregany M. Miniature ice detection sensor systems for aerospace applications. Micro Electro Mechanical Systems, 1998. MEMS 98. Proceedings, The Eleventh Annual International Workshop on 1998; 75-80.

3. Dutton B, Boonsang S, Dewhurst RJ. A new magnetic configuration for a small in-plane electromagnetic acoustic transducer applied to laser ultrasound measurements: modelling and validation. Sensors and Actuators A: Physical 2006; 125:249-259.

4. Boonsang S, Dutton B, Dewhurst RJ. Modelling of magnetic fields to enhance the performance of an in-plane EMAT for laser-generated ultrasound. Ultrasonics 2006; 44:657-665.

5. Murayama R, Mizutani K. Conventional electromagnetic acoustic transducer development for optimum Lamb wave modes. Ultrasonics 2002; 40:491-495.

6. Silva MZ, Gouyon R, Lepoutre F. Hidden corrosion detection in aircraft aluminium structures using laser ultrasonics and wavelet transform signal analysis. Ultrasonics 2003; 41:301-305.

7. Cosenza C, Kenderian S, Djordjevic BB, Green RE Jr, Pasta A. Generation of narrowband antisymmetric Lamb waves using a formed laser source in the ablative regime. IEEE Transactions on Ultrasonics, Ferroelectrics, and Frequency Control 2007; 54(1):147-156.

8. Staszewski WJ, Lee BC, Mallet L, Scarpa F. Structural health monitoring using scanning laser vibrometry: I. Lamb wave sensing. Smart Materials and Structures 2004; 13:251-260.

9. Lee BC, Staszewski WJ. Lamb wave propagation modelling for damage detection: II. Damage monitoring strategy. Smart materials and structures 2007; 16:260-274.

10. Bent AA, Hagood NW. Piezoelectric fiber composites with interdigitated electrodes. Journal of Intelligent Material Systems and Structures 1997; 11(8):903-919. 
11. Brunner AJ, Barbezat M, Huber C, Flueler PH. The potential of active fiber composites made from piezoelectric fibers for actuating and sensing applications in structural health monitoring. Materials and Structures 2005; 38:561-567.

12. Gentilman R, McNeal K, Schmidt G, Pizzochero A, Rosetti G Jr. Enchanced performances active fibercomposites. Smart Structures and Materials: Industrial and Commercial Applications of Smart Structures Technologies 2003; 5054:350-359.

13. Birchmeier M, Gsell D, Juon M, Brunner AJ, Paradies R, Dual J. Active fiber composites for the generation of Lamb waves. Ultrasonics 2009; 49:73-82.

14. Williams BR, Park G, Inman DJ, Wilkie KW. An overview of composite actuators with piezoceramic fibers. 20th International Modal Analysis Conference. Los Angeles 2002; 421-427.

15. High JW, Wilkie KW. Method of fabricating NASA-standard macro-fiber composite piezoelectric actuator 2003; NASA/TM-2003-212427 ARL-TR-2833.

16. Salas KI, Cesnik CES. Guided wave excitation by a CLoVER transducer for structural health monitoring: theory and experiments. Smart Material and Structures 2009; 18:1-27.

17. Salas KI, Cesnik CES. Design and characterization of the CLoVER transducer for structural health monitoring. Proceedings of SPIE 2008; 6935.

18. Howard MM, Lanza Di Scalea F. Macro-fiber composite piezoelectric rosettes for acoustic source location in complex structures. Smart Materials and Structures 2007; 16:1489-1499.

19. Thien AB, Chiamori HC, Ching JT, Wait JR, Park G. The use of macro-fibre composites for pipeline structural health assessment. Structural Control and Health Monitoring 2008; 15:43-63.

20. Mamishev AV, Sundara-Rajan K, Yang F, Du Y, Zahn M. Interdigital sensors and transducers. Proceedings of the IEEE 2004; 92(5):808-845.

21. Jin J, Quek ST, Wang Q. Design of interdigital transducers for crack detection in plates. Ultrasonics 2005; 43:481-493.

22. Monkhouse RSC, Wilcox PD, Cawley P. Flexible interdigital PVDF transducers for the generation of Lamb waves in structures. Ultrasonics 1997; 35:489-498.

23. Monkhouse RSC, Wilcox PW, Lowe MJS, Dalton RP, Cawley P. The rapid monitoring of structures using interdigital Lamb. Smart Materials and Structures 2000; 9:304-309.

24. Badcock RA, Birt EA. The use of 0-3 piezocomposite embedded Lamb wave sensors for detection of damage in advanced fibre composites. Smart Materials and Structures 2000; 9:291-297.

25. Diamanti K, Hodgkinson JM, Soutis C. Damage detection of composite laminates using PZT generated Lamb waves. Proceedings of the First European Workshop on Structural Health Monitoring. Paris 2002; 398-405.

26. Dimitriadis EK, Fuller CR, Rogers CA. Piezoelectric actuators for distributed vibration excitation of thin plates. Journal of Vibration and Acoustics 1991; 113:100-107.

27. Schulz MJ, Pai PF, Imman DJ. Health monitoring and active control of composite structures using piezoceramic patches. Composites, Part B 1999; 30:713-725.

28. Klepka A, Ambrozinski Ł. Selection of piezoceramic sensor parameters for damage detection and localization system. Diagnostyka/Polskie Towarzystwo Diagnostyki Technicznej 2010; 4:17-22.

29. Collet M, Ruzzene M, Cunefare KA. Generation of Lamb waves through surface mounted macro-fiber composite transducers. Smart Materials and Structures 2011; 20:1-14.

30. Ochoński J, Ambroziński Ł, Klepka A, Uhl T, Stępiński T. Choosing an appropriate sensor for the designed SHM system based on Lamb waves propagation. 11th IMEKO TC 10 Workshop on Smart Diagnostics of Structures, Krakow 2010.

31. Farrar CR, Park G, Allen DW, Todd MD. Sensor network paradigms for structural health monitoring. Structural Control and Health Monitoring 2006; 13:210-225.

32. Yu L, Cheng L, Su Z. Correlative sensor array and its applications to identification of damage in plate-like structures. Structural Control and Health Monitoring 2011, published online. DOI: 10.1002/stc.461

33. Manka M, Rosiek M, Martowicz A, Uhl T, Stepinski T. Design and simulations of interdigital transducers for Lamb-wave based SHM systems. Proceedings of 11th IMEKO TC 10 Workshop on Smart Diagnostics of Structures, Krakow 2010.

34. Manka M, Rosiek M, Martowicz A, Uhl T, Stepinski T. properties of interdigital transducers for Lamb-wave based SHM systems. The 8th International Workshop on Structural Health Monitoring-Stanford University 2011; 1488.

35. Bellan F, Bulletti A, Capineri L, Masotti L, Yaralioglu GG, Degertekin LF, Khuri-Yakub BT, Guasti F, Rosi E. A new design and manufacturing process for embedded Lamb waves interdigital transducers based on piezopolymer film. Sensors and Actuators A 2005; 123-124:379-387.

36. Capineri L, Gallai A, Masotti L, Materassi M. Design criteria and manufacturing technology of piezo-polymer transducer arrays for acoustic guided waves detection. IEEE Ultrasonic Symposium 2002.

37. Wilcox PD, Cawley P, Lowe MJS. Acoustic fields from PVDF interdigital transducers. IEE Proceedings Science, Measurement and Technology 1998; 145(5):250-259.

38. Luginbuhl P, Collins SD, Racine G, Gretillat MA, de Rooij NF, Brooks KG, Setter N. Microfabricated Lamb wave device based on PZT Sol-gel thin film for mechanical transport of solid particles and liquids. Journal of Microelectromechanical Systems 1997; 6(4):337-346.

39. Hayward G, Hailu B, Farlow R, Gachagan A, McNab A. The design of embedded transducers for structural health monitoring applications. Proceedings of SPIE 2001; 4327:312-323.

40. Na JK, Blackshire JL, Kuhra S. Design, fabrication and characterization of single-element interdigital transducers for NDT applications. Sensors and Actuators A 2008; 148:359-365.

41. Na JK, Blackshire JL. Interaction of Rayleigh surface waves with a tightly closed fatigue crack. NDT\&E International 2010; 43:432-439.

42. Box GEP, Draper NR. Empirical Model Building and Response Surfaces. John Wiley \& Sons Inc: New York, 1986.

43. Myers RH, Montgomery DC. Response Surface Methodology Process and Product Optimization Using Designed Experiments. John Wiley \& Sons Inc.: New York, 1995. 